\title{
Electrophysiological Abnormalities in VLCAD Deficient hiPSC-Cardiomyocytes Can Be Improved by Lowering Accumulation of Fatty Acid Oxidation Intermediates
}

\author{
Suzan J. G. Knottnerus ${ }^{1,2,+}$ (D) Isabella Mengarelli ${ }^{3, \dagger}{ }^{\dagger}$ Rob C. I. Wüst ${ }^{1}$, Antonius Baartscheer ${ }^{3}$, \\ Jeannette C. Bleeker ${ }^{1,2}{ }^{1}$, Ruben Coronel $^{3}$, Sacha Ferdinandusse ${ }^{1}$, Kaomei Guan ${ }^{4}{ }^{\circledR}$, \\ Lodewijk IJlst ${ }^{1}$, Wener $\mathrm{Li}^{4}{ }^{4}$, Xiaojing Luo ${ }^{4}$, Vincent M. Portero ${ }^{3}{ }^{(\mathbb{D}}$, Ying Ulbricht ${ }^{4}$, \\ Gepke Visser ${ }^{1,2}$, Ronald J. A. Wanders ${ }^{1}$, Frits A. Wijburg ${ }^{5}$, Arie O. Verkerk ${ }^{3,6}{ }^{\circledR}$, \\ Riekelt H. Houtkooper $1, * \mathbb{D}$ and Connie R. Bezzina ${ }^{3, *}$ \\ 1 Laboratory Genetic Metabolic Diseases, Amsterdam UMC, University of Amsterdam, \\ Amsterdam Gastroenterology and Metabolism, Amsterdam Cardiovascular Sciences, \\ 1105 AZ Amsterdam, The Netherlands; s.j.knottnerus@amsterdamumc.nl (S.J.G.K.); r.wust@vu.nl (R.C.I.W.); \\ jeannette.bleeker@gmail.com (J.C.B.); s.ferdinandusse@amsterdamumc.nl (S.F.); \\ l.ijlst@amsterdamumc.nl (L.I.); G.Visser-4@umcutrecht.nl (G.V.); r.j.wanders@amc.uva.nl (R.J.A.W.) \\ 2 Department of Paediatric Metabolic Diseases, Wilhelmina Children's Hospital, \\ University Medical Center Utrecht, 3584 EA Utrecht, The Netherlands \\ 3 Department of Clinical and Experimental Cardiology, Heart Center, Amsterdam Cardiovascular Sciences, \\ Amsterdam UMC, University of Amsterdam, 1105 AZ Amsterdam, The Netherlands; \\ i.mengarelli@amsterdamumc.nl (I.M.); a.baartscheer@amsterdamumc.nl (A.B.); \\ r.coronel@amsterdamumc.nl (R.C.); v.m.portero@amsterdamumc.nl (V.M.P.); \\ a.o.verkerk@amsterdamumc.nl (A.O.V.) \\ 4 Institute of Pharmacology and Toxicology, Technische Universität Dresden, 01069 Dresden, Germany; \\ kaomei.guan@tu-dresden.de (K.G.); wener.li@tu-dresden.de (W.L.); xiaojing.luo@tu-dresden.de (X.L.); \\ yingulbricht@gmail.com (Y.U.) \\ 5 Department of Paediatric Metabolic Diseases, Emma Children's Hospital, Amsterdam UMC, \\ University of Amsterdam, 1105 AZ Amsterdam, The Netherlands; f.a.wijburg@amsterdamumc.nl \\ 6 Department of Medical Biology, Amsterdam Cardiovascular Sciences, Amsterdam UMC, \\ University of Amsterdam, 1105 AZ Amsterdam, The Netherlands \\ * Correspondence: r.h.houtkooper@amsterdamumc.nl (R.H.H.); c.r.bezzina@amsterdamumc.nl (C.R.B.) \\ + These authors contributed equally to this work.
}

Received: 12 March 2020; Accepted: 5 April 2020; Published: 8 April 2020

\begin{abstract}
Patients with very long-chain acyl-CoA dehydrogenase deficiency (VLCADD) can present with life-threatening cardiac arrhythmias. The pathophysiological mechanism is unknown. We reprogrammed fibroblasts from one mildly and one severely affected VLCADD patient, into human induced pluripotent stem cells (hiPSCs) and differentiated these into cardiomyocytes (VLCADD-CMs). VLCADD-CMs displayed shorter action potentials (APs), more delayed afterdepolarizations (DADs) and higher systolic and diastolic intracellular $\mathrm{Ca}^{2+}$ concentration $\left(\left[\mathrm{Ca}^{2+}\right]_{\mathrm{i}}\right)$ than control CMs. The mitochondrial booster resveratrol mitigated the biochemical, electrophysiological and $\left[\mathrm{Ca}^{2+}\right]_{\mathrm{i}}$ changes in the mild but not in the severe VLCADD-CMs. Accumulation of potentially toxic intermediates of fatty acid oxidation was blocked by substrate reduction with etomoxir. Incubation with etomoxir led to marked prolongation of AP duration and reduced DADs and $\left[\mathrm{Ca}^{2+}\right]_{\mathrm{i}}$ in both VLCADD-CMs. These results provide compelling evidence that reduced accumulation of fatty acid oxidation intermediates, either by enhanced fatty acid oxidation flux through increased mitochondria biogenesis (resveratrol) or by inhibition of fatty acid transport into the mitochondria (etomoxir), rescues pro-arrhythmia defects in VLCADD-CMs and open doors for new treatments.
\end{abstract}


Keywords: VLCADD; arrhythmias; hiPSC; acylcarnitines

\section{Introduction}

Very long-chain acyl-CoA dehydrogenase deficiency (VLCADD) (OMIM 201475), an autosomal recessive inborn error of metabolism, caused by pathogenic mutations in the ACADVL gene, is a mitochondrial long-chain fatty acid oxidation (lcFAO) disorder [1,2]. Patients with VLCADD may develop hypoglycemia, rhabdomyolysis, cardiomyopathy and cardiac arrhythmias [3-6]. Because some of these clinical signs and symptoms can be fatal early in life and are at least partly preventable, VLCADD has been included in newborn screening programs worldwide [7]. There is a marked variety in the development of clinical symptoms among patients [8]. Cardiomyopathy and rhythm disturbances occur almost exclusively in patients with a low residual lcFAO flux $(<10 \%)$ and are not prevented by early dietary treatment (avoidance of fasting) [7]. Mechanisms underlying cardiac arrhythmias in VLCADD, which can occur even in the absence of structural cardiac malformation $[4,9]$ are not well understood. Two putative pathophysiological mechanisms have been proposed for the cardiac phenotype-(1) energy shortage due to deficient adenosine triphosphate (ATP) synthesis from lcFAO, and (2) accumulation of lcFAO intermediates including long-chain acylcarnitines (LCACs) $[9,10]$. Of note, in acute myocardial ischemia, rapid LCAC accumulation also occurs [11] and for this reason the modulating effects of LCACs on cardiac action potentials (APs) and ion currents have been widely studied [12-17]. Exogenously added LCACs can induce a variety of electrophysiological alterations in ventricular myocytes, including AP shortening [13], cellular uncoupling [18] and delayed afterdepolarizations (DADs) $[13,14]$, the latter representing an important cellular mechanism for arrhythmias $[19,20]$.

Previous work showed that resveratrol increases lcFAO flux and decreases LCAC accumulation in fibroblasts of VLCADD patients with milder phenotypes [21,22]. In these studies, the effects of resveratrol were thought to be mediated by an increase in the amount of mutated VLCAD protein via sirtuin 1 activation [21]. In cells with null-type mutations (e.g., nonsense, frameshift, truncating mutations) in $A C A D V L$, resveratrol cannot increase lcFAO flux, considering the virtually absent VLCAD activity [21,23]. Decrease in potentially toxic LCAC accumulation can also be acquired by incubating cells with etomoxir, a pharmacological inhibitor of carnitine palmitoyltransferase 1 (CPT1) [24], one of the enzymes that is involved in the mitochondrial import of long-chain fatty acids by converting long-chain acyl-CoAs to LCACs [25].

Development of human induced pluripotent stem cell (hiPSC) technology provides the opportunity to generate VLCADD patient-specific cardiomyocytes (VLCADD-CMs) and to study diseases on a cell-type specific level [26]. In order to progress towards prevention or therapeutic interventions for VLCADD-induced cardiac arrhythmias, we generated hiPSC-CMs from two patients harboring distinct $A C A D V L$ genetic defects and studied the underlying mechanism of the pro-arrhythmic phenotype using compounds affecting different aspects of lcFAO biochemistry, namely resveratrol and etomoxir.

\section{Results}

\subsection{VLCADD Patients}

The biochemical and clinical characteristics of the patients are described in Table 1. Patient 1 had a residual lcFAO flux in fibroblasts that was $32 \%$ of control values suggesting a mild phenotype. She presented for the first time at the age of 15 months with severe hypoglycemia $(0.2 \mathrm{mmol} / \mathrm{L})$ and hypothermia $\left(35.9^{\circ} \mathrm{C}\right)$, during an infection. ECGs were not obtained during this episode. At age 15 years, a routine Holter-ECG showed no abnormalities. With dietary adjustments, that is, avoidance of fasting, no severe episodes occurred after the initial presentation. Patient 2 had a lcFAO flux of $7 \%$, indicative of a severe VLCADD phenotype. She presented at the age of 6 weeks with 
hypoglycemia $(1.7 \mathrm{mmol} / \mathrm{L})$, convulsions and tachycardia. At age of 2 months, echocardiography revealed a hypertrophic cardiomyopathy. During childhood, she had frequent metabolic derangements and episodes of relapsing cardiomyopathy. No ECGs during metabolic derangement were available. In a stable condition at age 15 years, the patient showed aspecific repolarization abnormalities on the ECG (flat ST-T segments in the inferior and left lateral leads) and early repolarizations in the inferior leads. A routine Holter-ECG at age 18 years showed single atrial and ventricular ectopy and a Mobitz II AV-block during sleep.

Table 1. Clinical and biochemical characteristics of patients.

\begin{tabular}{|c|c|c|}
\hline PID (Name of hiPSC Line) & Patient 1 (iVLCADD1) & Patient 2 (iVLCADD2) \\
\hline Age of presentation (current age) & 1.25 y (23 y) & 0.1 y (22 y) \\
\hline Sex & female & female \\
\hline \multirow{2}{*}{ Mutations in $A C A D V L$ gene } & c. $848 \mathrm{~T}>\mathrm{C}$ (p.Val283Ala) & c.104delC (p.Pro35Leufs*26) \\
\hline & c.1141_1143delGAG (p.Glu381del) & c.104delC (p.Pro35Leufs*26) \\
\hline VLCAD activity & Fibroblasts: $0.10(3 \%)$ & Fibroblasts: $<0.06(0 \%)$ \\
\hline $\begin{array}{l}\text { (in } \mathrm{nmol} / \mathrm{min} / \mathrm{mg} \text { and } \% \text { of } \\
\text { controls) }\end{array}$ & Lymphocytes: $0.18(5 \%)$ & Lymphocytes $<0.15(0 \%)$ \\
\hline $\begin{array}{l}\text { lcFAO flux } \\
(\% \text { of control })\end{array}$ & (\% of control) & 7 \\
\hline Maximal Creatine Kinase & $400 \mathrm{U} / \mathrm{L}$ & $99889 \mathrm{U} / \mathrm{L}$ \\
\hline Signs at presentation & $\begin{array}{c}\text { Hypoglycemia } 0.2 \mathrm{mmol} / \mathrm{L} \\
\text { Hypothermia }\left(35.9^{\circ} \mathrm{C}\right)\end{array}$ & $\begin{array}{l}\text { Hypoglycemia } 1.7 \mathrm{mmol} / \mathrm{L} \text {, vomiting, } \\
\text { convulsions, cardiomyopathy (reversible) }\end{array}$ \\
\hline \multirow[t]{2}{*}{ Cardiac history } & $\begin{array}{l}\text { Age 15y: Holter ECG: Normal } \\
\text { conduction. }\end{array}$ & $\begin{array}{l}\text { Age 14y: ECG: aspecific repolarization } \\
\text { abnormalities. (flat ST-T segments in the } \\
\text { inferior and left lateral leads). Some early } \\
\text { repolarizations in the inferior leads. } \\
\text { Age 15y: dilated cardiomyopathy } \\
\text { (reversible) }\end{array}$ \\
\hline & & $\begin{array}{c}\text { Age 18y: Holter ECG: single ectopy from } \\
\text { the atrial side as well as from the } \\
\text { ventricular side. Mobitz II AV-block } \\
\text { during sleep. }\end{array}$ \\
\hline Other signs or symptoms & None & Rhabdomyolysis \\
\hline & & Exercise intolerance \\
\hline
\end{tabular}

\subsection{Generation of Patient-Specific VLCADD-CM}

Fibroblasts from both patients and from a healthy control were reprogrammed to generate hiPSC lines (iVLCADD1 from Patient 1, iVLCADD2 from Patient 2 and iCTRL (Figure S1)). These lines were differentiated to cardiomyocytes (iVLCADD1-CMs, iVLCADD2-CMs and iCTRL-CMs; details in Material and Methods) and subjected to biochemical analyses as well as single cell electrophysiological and $\left[\mathrm{Ca}^{2+}\right]_{\mathrm{i}}$ analysis.

LcFAO flux is expressed as percentage of the mean flux in healthy control cell lines measured in the same experiment. VLCAD activities are expressed as percentage of reference mean. Detection limit for VLCAD activity in lymphocytes and fibroblasts was $0.15 \mathrm{nmol} / \mathrm{min} / \mathrm{mg}$ and $0.06 \mathrm{nmol} / \mathrm{min} / \mathrm{mg}$, respectively. ECG: electrocardiogram. lcFAO flux: long-chain fatty acid oxidation flux. Maximal creatine kinase is the highest value reported in the medical history of the patient. 


\subsection{VLCADD-CMs Accumulate Long-Chain Acylcarnitine Species}

To confirm the lcFAO deficiency in the VLCADD hiPSC-CMs, we measured LCAC accumulation in the medium after supplementation with stable isotope-labeled $\left[\mathrm{U}-{ }^{13} \mathrm{C}\right]$-palmitic acid, as a diagnostic marker for VLCADD. The ratio of LCACs over C2-carnitine was markedly increased in the incubation medium of both iVLCADD1-CMs and iVLCADD2-CMs (Figure 1A), confirming that these cells retained their VLCAD deficiency. We also tested if cells accumulated LCAC under regular hiPSC-CM culture conditions. These cells were routinely cultured in medium containing a relatively low amount of carnitine and fatty acids $(\approx 11 \mu \mathrm{mol} / \mathrm{L}$ and $\approx 30 \mu \mathrm{mol} / \mathrm{L}$ respectively) but even under these conditions LCAC levels were increased in VLCADD-CMs compared to iCTRL-CMs (Figure S2).
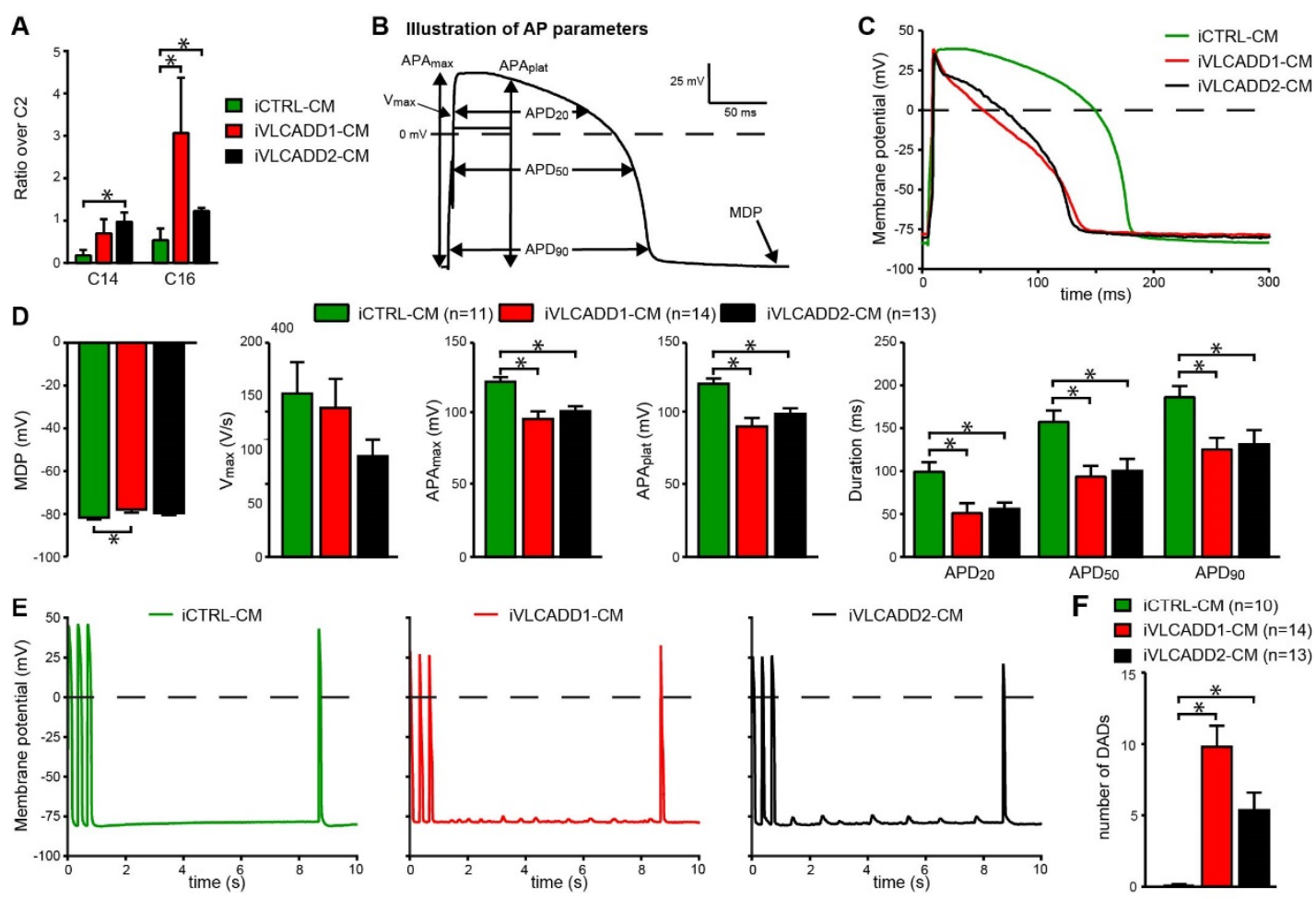

F $\square$ ICTRL-CM $(n=10)$

口 iVLCADD1-CM ( $n=14)$ - iVLCADD2-CM ( $(\mathrm{n}=13)$
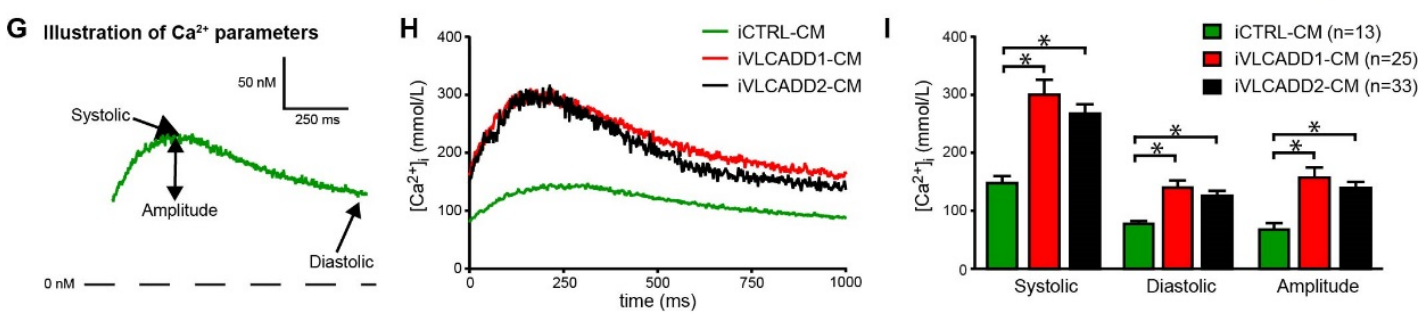

Figure 1. Electrophysiological abnormalities in very long-chain acyl-CoA dehydrogenase deficiency (VLCADD) human induced pluripotent stem cell- cardiomyocytes (hiPSC-CMs). (A) Increased C14/C2 carnitine and C16/C2 carnitine ratio in medium of iVLCADD1-CMs and iVLCADD2-CMs compared to control cardiomyocytes (iCTRL-CM) after $96 \mathrm{~h}$ culturing with palmitate loading. Data expressed as mean + SD $(n=3)$. Statistical analysis was performed using a two-way ANOVA with Sidaks multiple comparisons test. ${ }^{*}=p<0.05$. (B) Analyzed action potential (AP) parameters. (C) Representative APs paced at $1 \mathrm{~Hz}$. (D) Average AP parameters. (E) Typical delayed afterdepolarizations (DADs) measured during an $8 \mathrm{~s}$ pause after fast pacing $(3 \mathrm{~Hz})$. After this pause, a single AP was evoked to confirm that no early afterdepolarizations occurred. (F) Average number of DADs. Electrophysiology data are expressed as mean + SEM of individual measured cells $(n=11-13)$. (G) Analyzed parameters in $\mathrm{Ca}^{2+}$ transients. (H) Representative $\mathrm{Ca}^{2+}$ transients of Indo- 1 loaded hiPSC-CMs paced at $1 \mathrm{~Hz}$. (I) Average $\left[\mathrm{Ca}^{2+}\right] \mathrm{i}($ mean $+\mathrm{SEM}){ }^{*}=p<0.05$. Statistical analysis was assessed with one-way ANOVA with Tukey's multiple comparisons test. 


\subsection{VLCADD-CMs Exhibit Shortened Action Potentials and Delayed after Depolarizations}

Next, we assessed the implications of VLCADD on various AP parameters (Figure 1B) and susceptibility to early afterdepolarization (EAD) and DAD generation in dissociated single hiPSC-CMs. Representative APs are shown in Figure $1 \mathrm{C}$ and average AP parameters are shown in Figure 1D. Maximal AP amplitude (APAmax), AP plateau amplitude (APAplat) and AP duration measured at $20 \%, 50 \%$ and $90 \%$ of repolarization $\left(\mathrm{APD}_{20}, \mathrm{APD}_{50}\right.$ and $\mathrm{APD}_{90}$, respectively) were significantly lower in the two VLCADD-CM lines compared to iCTRL-CMs (Figure 1D). The susceptibility to occurrence of DADs was tested by applying a fast pacing stimulus followed by an 8-s pause. After the 8-s pause, a single AP was evoked to test the occurrence of EADs. EADs were never observed, however, we observed many DADs in VLCADD-CMs. Figure 1E,F show typical traces and the average number of DADs, respectively. In both VLCADD-CMs the number of DADs was markedly increased compared to control (Figure 1F).

\subsection{VLCADD-CMs Show Increased $\mathrm{Ca}^{2+}$ Concentration}

Using fluorescence measurements, we characterized various $\mathrm{Ca}^{2+}$ transient parameters (Figure 1G) and found a higher systolic and diastolic intracellular $\mathrm{Ca}^{2+}$ concentration $\left(\left[\mathrm{Ca}^{2+}\right]_{\mathrm{i}}\right)$ as well as an increased transient amplitude in iVLCADD1-CMs and iVLCADD2-CMs compared to iCTRL-CMs (Figure 1H-I). Collectively, these results demonstrate that hiPSC-CMs from VLCADD patients exhibit increased LCAC accumulation and that $\left[\mathrm{Ca}^{2+}\right]_{\mathrm{i}}$ is increased, accompanied by AP shortening and presence of DADs.

\subsection{Resveratrol Improves Biochemical and Electrophysiological Derangements in VLCADD-CM of Patient 1}

In line with expectations based on genotype (Patient 1 is compound heterozygous for missense mutations which generate a mitochondrial enzyme with residual activity, whereas Patient 2 is homozygous for a truncating mutation which eliminates the enzyme activity), incubation with the mitochondrial booster resveratrol induced a strong increase in lcFAO flux in cultured fibroblasts from patient 1 but not in patient 2 (Figure S3). Therefore, we hypothesized that resveratrol would reduce LCAC accumulation and would mitigate the electrophysiological alterations only in iVLCADD1-CMs. Indeed, we found that LCAC accumulation was reduced in iVLCADD1-CMs but not in iVLCADD2-CMs (Figure $2 \mathrm{~A}$ ) after $48 \mathrm{~h}$ pre-incubation with resveratrol. Strikingly, resveratrol also increased the AP amplitude and prolonged the AP in iVLCADD1-CMs (Figure $2 \mathrm{~B}, \mathrm{C}$ ). In addition, $\left[\mathrm{Ca}^{2+}\right]_{\mathrm{i}}$ and the number of DADs was significantly reduced after resveratrol treatment in iVLCADD1-CMs but not iVLCADD2-CMs (Figure 2D-F). 
A

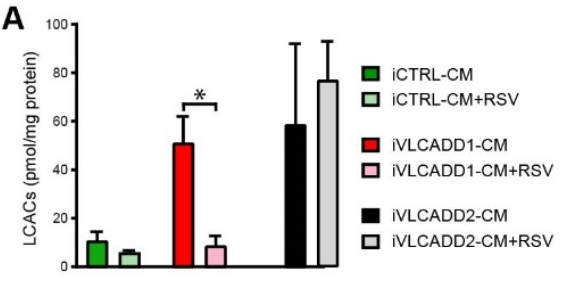

B

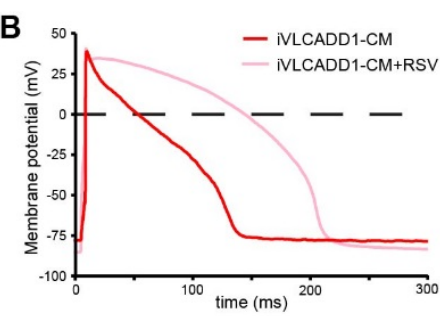
ㅁ iVLCADD1-CM $(n=14)$ iVLCADD2-CM (n=13)
iVLCADD1-CM+RSV (n=10) iVLCADD2-CM+RSV (n=7)

C
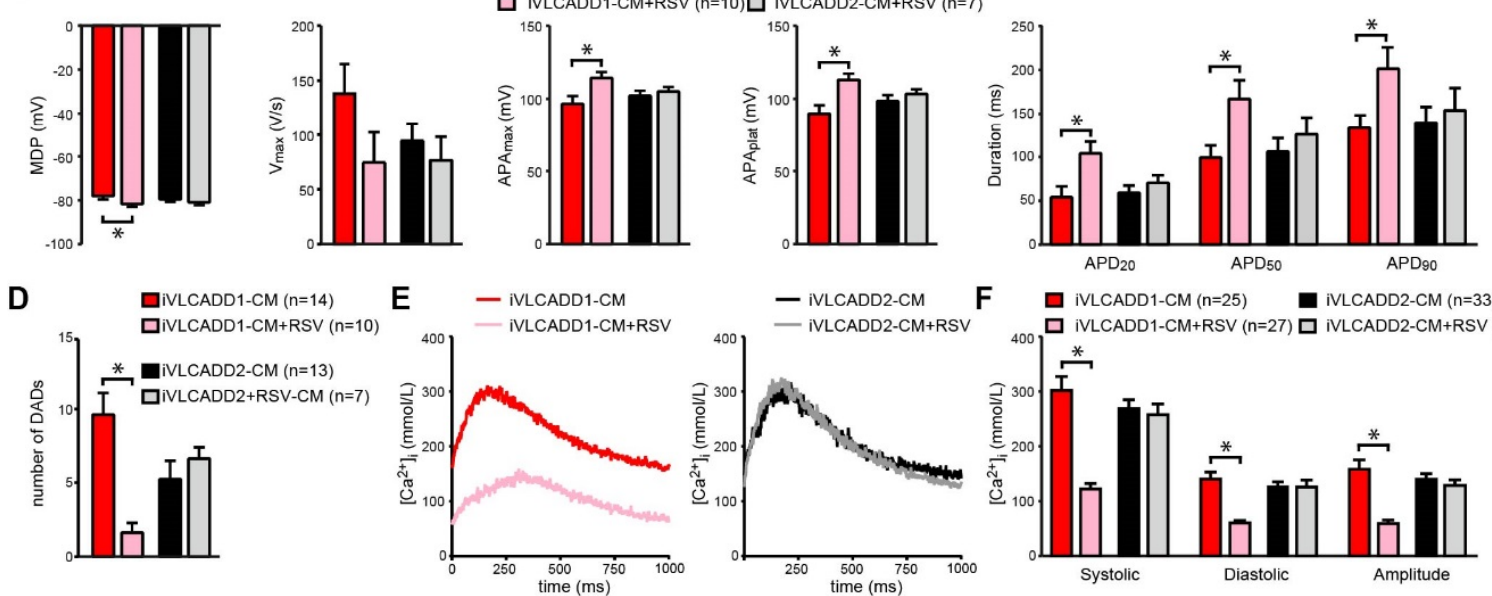

F $\square$ iVLCADD1-CM (n=25)

iVLCADD2-CM ( $n=33)$

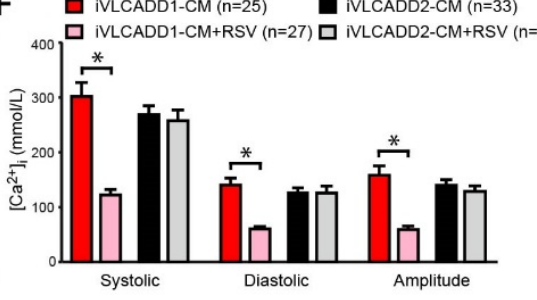

Figure 2. Rescue of electrophysiological abnormalities in iVLCADD1-CMs after pre-incubation with Resveratrol (RSV; $50 \mu \mathrm{mol} / \mathrm{L}$ ). (A) Intracellular acylcarnitine levels in iVLCADD1-CMs, iVLCADD2-CMs and iCTRL-CMs after RSV incubation for $96 \mathrm{~h}$. Sum of long-chain acylcarnitines (LCACs) (C12-, C14-, C14:1-, C16-, C16:1-, C18-, C18:1-carnitine). Values represent mean + SD in two biological replicates. Statistical analysis was performed using a two-way ANOVA with Tukey's multiple comparisons test. ${ }^{*}=p<0.05$. (B) Representative APs paced at $1 \mathrm{~Hz}$. (C) Average AP parameters after RSV or vehicle (dimethyl sulfoxide (DMSO)) pre-incubation. (D) Number of DADs in absence and presence of RSV. (E) Representative $\mathrm{Ca}^{2+}$ transients of Indo-1 loaded hiPSC-CMs paced at $1 \mathrm{~Hz}$ in presence of RSV. (F) Average $\left[\mathrm{Ca}^{2+}\right]_{\mathrm{i}}$ after RSV or vehicle (DMSO) pre-incubation. For AP analyses and $\left[\mathrm{Ca}^{2+}\right]_{\mathrm{i}}$ data is indicated as mean+SEM. ${ }^{*}=p<0.05$. Number of cells is indicated in the figure. Statistical analysis was assessed with one-way ANOVA with Tukey's multiple comparisons test.

2.7. Blocking of lcFAO Flux and Consequently Reducing Accumulation of lcFAO Intermediates Mitigated Electrophysiological Abnormalities in VLCADD-CM

The rescued electrophysiological phenotype by resveratrol in iVLCADD1-CMs but not iVLCADD2-CMs strongly suggests that the electrophysiological and $\left[\mathrm{Ca}^{2+}\right]_{i}$ improvement is consequent to improved lcFAO flux. In the next experiments we investigated the effect of lowering the LCAC accumulation without increase of the lcFAO flux on the AP characteristics and $\mathrm{Ca}^{2+}$-homeostasis. We used etomoxir to inhibit the production of LCAC by CPT1, which in parallel leads to a further block of the lcFAO flux. Pre-incubation with etomoxir for $48 \mathrm{~h}$ led to a complete reduction of intracellular LCAC accumulation in both iVLCADD1-CMs and iVLCADD2-CMs (Figure 3A). This reduction was combined with a concomitant increase in $\mathrm{AP}$ amplitudes and $\mathrm{APD}_{20}$ as well as a reduced occurrence of DADs (Figure 3B-D). Also the increase in $\left[\mathrm{Ca}^{2+}\right]_{\mathrm{i}}$ was reduced in both lines (Figure 3E,F). 
A
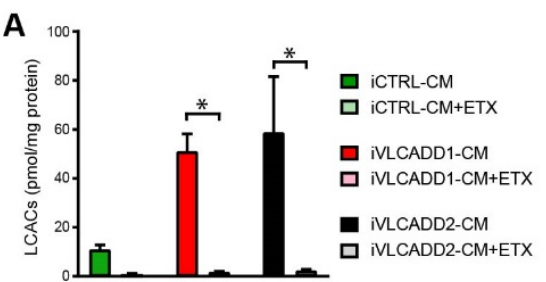
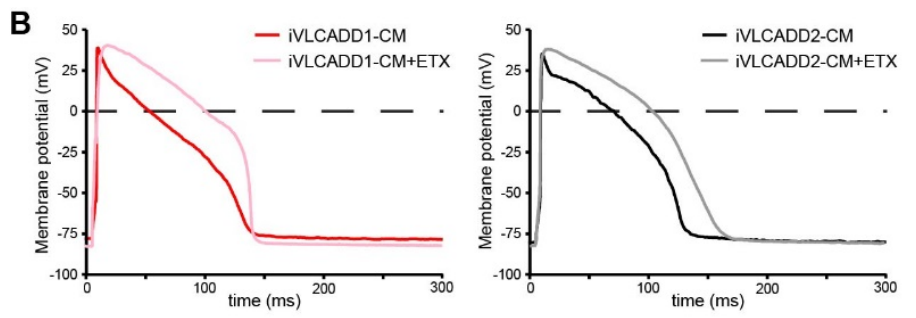

C
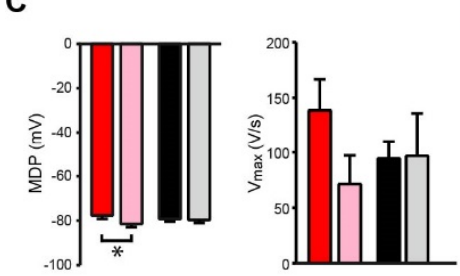

ㅁ $\mathrm{IVLCADD1}-\mathrm{CM}(\mathrm{n}=14)$

IVLCADD2-CM (n=13)

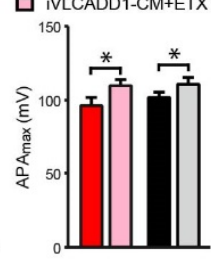

D
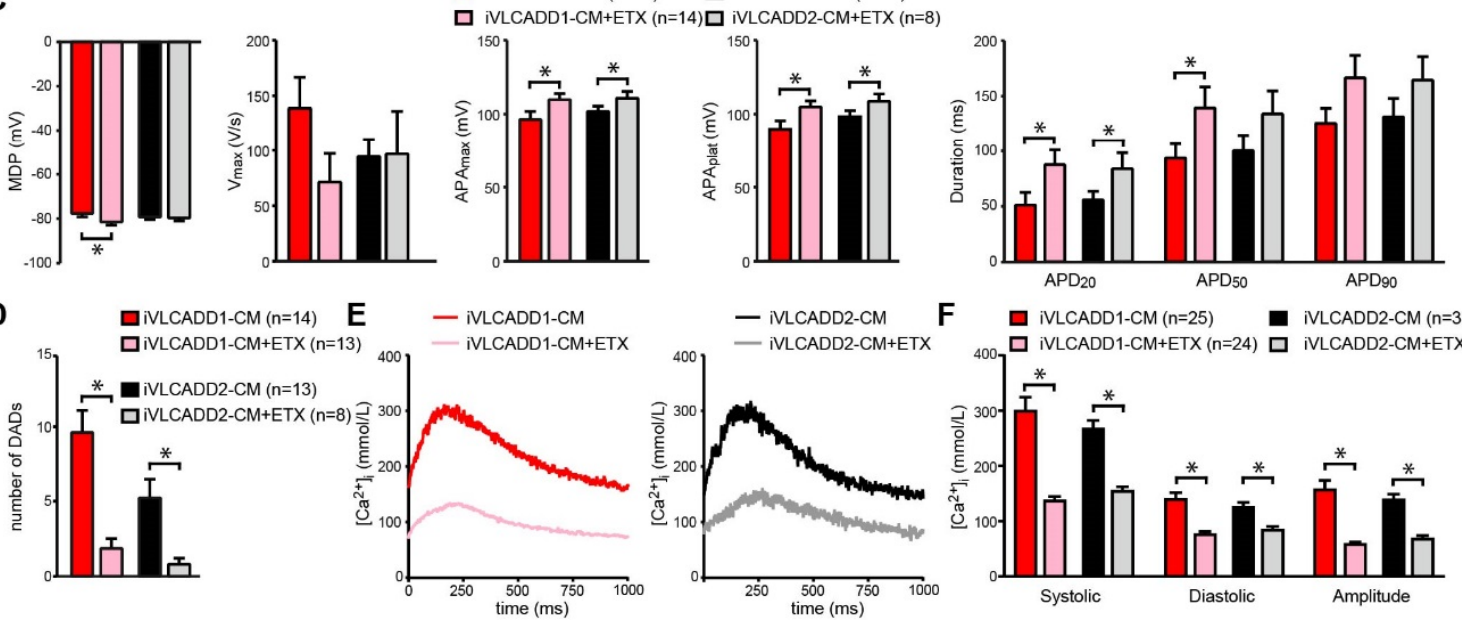

$\mathbf{F}$

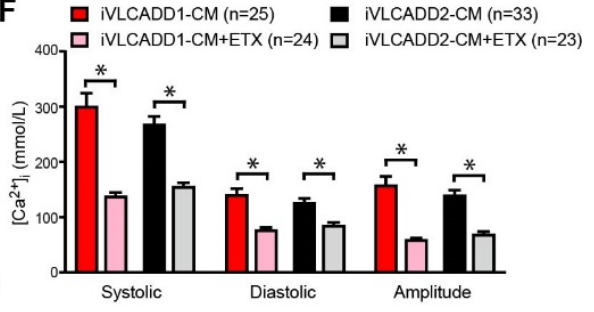

Figure 3. Rescue of electrophysiological abnormalities in iVLCADD1-CMs and iVLCADD2-CMs after pre-incubation with Etomoxir (ETX; $100 \mu \mathrm{mol} / \mathrm{L}$ ). (A) Intracellular acylcarnitine levels in iVLCADD1-CMs, iVLCADD2-CMs and iCTRL-CMs after ETX incubation for $96 \mathrm{~h}$. Sum of LCAC (C12-, C14-, C14:1-, C16-, C16:1-, C18-, C18:1-carnitine). Values represent mean + SD in two biological replicates of the different hiPSC-CM lines. Statistical analysis was performed using a two-way ANOVA with Tukey's multiple comparisons test. ${ }^{*}=p<0.05$. (B) Representative APs measured in iVLCADD1-CMs and iVLCADD2-CMs after pre-incubation with ETX or vehicle. (C) Average AP parameters after pre-incubation with ETX or vehicle. (D) Number of DADs in absence and presence of ETX. (E) Representative $\mathrm{Ca}^{2+}$ transients of Indo- 1 loaded hiPSC-CMs paced at $1 \mathrm{~Hz}$ in presence of ETX. (F) Average $\left[\mathrm{Ca}^{2+}\right] \mathrm{i}$ after ETX or vehicle pre-incubation. For AP analyses and $\left[\mathrm{Ca}^{2+}\right]_{\mathrm{i}}$ data is indicated as mean+SEM. ${ }^{*}=p<0.05$. Statistical analysis was assessed with one-way ANOVA with Tukey's multiple comparisons test.

\section{Discussion}

Here, we study hiPSC-derived cardiomyocytes of patients with VLCADD and examined two putative pathophysiological mechanisms that have been proposed for the cardiac phenotype: (1) energy shortage due a lower lcFAO flux, and, (2) accumulation of lcFAO intermediates. First, we found that VLCADD-CMs of two unrelated VLCADD patients displayed severe electrophysiological abnormalities compared to control CMs: a shortening in AP duration, a marked increase in DADs and an increased systolic and diastolic $\mathrm{Ca}^{2+}$ concentration. Secondly, improvement of all these electrophysiological derangements was possible by incubation with resveratrol, however only in iVLCADD1-CMs, in accordance with the increase of lcFAO flux by resveratrol in fibroblasts of this patient (only in Patient 1 and not in Patient 2), Thirdly, a reduction of lcFAO intermediates accumulation without increasing lcFAO flux, through counterintuitive substrate reduction therapy with etomoxir, was sufficient to restore the majority of the normal AP characteristics and $\left[\mathrm{Ca}^{2+}\right]_{i}$ in VLCADD-CMs. We hence conclude that accumulation of lcFAO intermediates is the mechanism underlying the disturbed AP characteristics and arrhythmia propensity observed in VLCADD.

In VLCADD-CMs derived from two unrelated patients we have documented severe electrophysiological abnormalities (AP shortening, DADs) that likely explain, at least in part, the cardiac arrhythmias observed in VLCADD patients. DADs are caused by spontaneous $\mathrm{Ca}^{2+}$ release 
from the sarcoplasmic reticulum, which typically occurs in $\mathrm{Ca}^{2+}$-overload conditions [27]. The higher diastolic $\left[\mathrm{Ca}^{2+}\right]$ i we observed in VLCADD-CMs is consistent with the increased occurrence of DADs, while the higher systolic $\left[\mathrm{Ca}^{2+}\right]_{\mathrm{i}}$ may be an explanation for the observed AP shortening via increased calcium-induced L-type $\mathrm{Ca}^{2+}$ current $\left(\mathrm{I}_{\mathrm{Ca}, \mathrm{L}}\right)$ inactivation and increased $\mathrm{K}^{+}$currents [28,29]. In addition, it was previously shown that intracellularly delivered LCACs suppress the $\mathrm{I}_{\mathrm{Ca}, \mathrm{L}}$ density [30], which may further contribute to the found AP shortening. AP shortening facilitates the maintenance of reentrant arrhythmias [31] and DADs may cause triggered activity [19,32,33].

VLCADD patients can have a very heterogeneous clinical outcome [7]. Fibroblasts from Patient 1 in our study exhibited a residual lcFAO flux of $32 \%$ of control values, a cellular evaluation that could agree with the development of a mild phenotype [7,34]. In line with this, Patient 1 , never presented with a cardiac phenotype or ECG abnormalities throughout life, although she followed since diagnosis a diet with limited periods of fasting. Still, the iVLCADD1-CMs showed severe electrophysiological derangements. A reason for this might be that hiPSC-CMs are known to present with a still immature phenotype under many aspects, therefore these cells may have a less developed mitochondrial system for FAO flux compared to adult cardiomyocytes [35]. Considering this, in the VLCADD-CMs the lcFAO flux may be relatively low due to both immaturity of the cells in combination with the ACADVL mutation. Perhaps in the immature hiPSC-CMs, this combination of events causes a more severe phenotype in single hiPSC-CMs than that observed in these individual patients.

In iVLCADD1-CMs both AP abnormalities and the derangements in $\mathrm{Ca}^{2+}$ homeostasis were prevented completely by resveratrol. The effects of resveratrol on lcFAO flux are the result of an increased amount of mutated VLCAD protein with residual activity in VLCADD fibroblasts [21]. With this in mind, it was expected that resveratrol would have had no effect effects on LCAC accumulation or electrophysiological parameters in iVLCADD2-CM (homozygous for a deletion mutation with no residual activity).

Incubation with etomoxir resulted in complete reduction of LCAC concentration in both VLCADD-CM lines. Without LCAC production by CPT1, lcFAO flux will be completely blocked, since transport of long-chain acyl-CoAs to the mitochondria is hampered. Still, the AP characteristics as well as $\left[\mathrm{Ca}^{2+}\right]_{\mathrm{i}}$ homeostasis were improved. These observations suggest that lcFAO intermediate accumulation (i.e., LCACs or intramitochondrial long-chain acyl-CoAs) underlies the pro-arrhythmia defects in VLCADD-CMs. This is further supported by previous studies in which exogenous addition of large amounts of LCACs to isolated hearts led to anomalies in the electrophysiological properties of cardiomyocytes $[12,15]$.

Our data also provide potential explanations for the hypertrophic cardiomyopathy that is observed in some patients. Indeed, elevated $\left[\mathrm{Ca}^{2+}\right]_{\mathrm{i}}$ is involved in the development of hypertrophic cardiomyopathy caused by sarcomere mutations $[36,37]$. It is hence tempting to speculate that the disturbed $\left[\mathrm{Ca}^{2+}\right]_{i}$ homeostasis we observed in VLCADD-CMs may underlie the cardiomyopathy in patients with VLCADD.

A potential limitation of our study is that resveratrol and etomoxir also have acute effects on cardiac electrophysiology and $\mathrm{Ca}^{2+}$ homeostasis $[29,38]$. However, in our study the cells were pre-incubated and resveratrol and etomoxir were absent during measurement, therefore excluding direct and acute effects on molecules regulating cardiac electrophysiology and $\mathrm{Ca}^{2+}$ homeostasis. In agreement with this, we only observed an effect of resveratrol in iVLCADD1-CM and not iVLCADD2-CM, which would have been expected if the effect of resveratrol were independent of VLCADD.

Based on our findings we conclude that accumulation of lcFAO intermediates is important in development of arrhythmias in VLCADD, hence a strategy to prevent lcFAO intermediate accumulation in patients is required. One of the current treatment strategies of lcFAODs is replacing dietary long-chain triglycerides (LCT) with medium-chain length triglycerides (MCT). Theoretically this would lead to less lcFAO intermediate production, since the enzymes responsible for medium chain fatty acid oxidation are functioning normally in individuals with lcFAODs. However, it should be noted that contrasting data have been reported on the effects of MCT on LCAC accumulation. On the one 
hand, a significant post-exercise lowering of LCACs in plasma was found after MCT in children with lcFAOD [39]. On the other hand, other studies show that in lcFAOD mice [40], human lcFAOD fibroblasts [41] and in premature infants without a lcFAOD [42], MCT can be elongated to LCT and even lead to higher LCAC accumulation [43]. Taken together these studies suggest that dietary modification with MCT in lcFAODs disorders has to be carefully adapted to energy demand in order to prevent lcFAO intermediate accumulation.

In future studies it would be interesting to test the effect of medium-chain fatty acid supplementation to the culture medium of VLCADD-CMs as well as supplementation of other current treatment modalities such as carnitine and triheptanoin [2] on the electrophysiological derangements in VLCADD-CM.

In conclusion, we provide insight into cardiomyocyte electrophysiological abnormalities underlying VLCADD and provide compelling evidence that pharmacologically reduced accumulation of lcFAO intermediates, either by enhancement (resveratrol) or blockage (etomoxir) of lcFAO flux, rescues the cellular arrhythmic phenotype in VLCADD cardiomyocytes, also in cells of a patient with a very severe VLCADD. This finding potentially impacts on the treatment of patients with VLCADD.

\section{Materials and Methods}

\subsection{Patient Selection and Clinical Data Analysis}

Two patients with genetically confirmed VLCADD were recruited at the Dutch FAO Expertise Center in Utrecht. Primary fibroblasts were already available for diagnostics. Written informed consent for the use of patient data and fibroblasts was obtained from both patients.

\subsection{Fibroblast Culture}

Fibroblasts derived from VLCADD patients and healthy controls were maintained in a humidified atmosphere containing 5\% $\mathrm{CO}_{2}$ in HAM F-10 medium (Gibco) supplemented with $10 \%$ fetal bovine serum (Gibco) and antibiotics $(100 \mu \mathrm{g} / \mathrm{mL}$ streptomycin, $100 \mathrm{U} / \mathrm{mL}$ penicillin and $250 \mu \mathrm{g} / \mathrm{mL}$ amphotericin) [44].

\subsection{VLCAD Activity}

VLCAD activity in lymphocytes and fibroblasts was measured using palmitoyl-CoA as substrate as described previously [25]. Data is expressed as nmol per minute per mg protein and percentage of reference mean that was valid at time of analysis.

\subsection{Measurement of Long-Chain Fatty Acid Oxidation (lcFAO) Flux}

Skin fibroblast were plated in 48-well plates. Long-chain fatty acid oxidation was measured by the production of ${ }^{3} \mathrm{H}_{2} \mathrm{O}$ from [9- ${ }^{3} \mathrm{H}(\mathrm{N})$ ]-oleic acid as described previously [45]. Measurements were done at $37^{\circ} \mathrm{C}$ in duplicate and lcFAO flux is expressed as percentage of mean activity of skin fibroblasts of healthy controls measured in the same experiment.

\subsection{Acylcarnitine Profiling}

Fibroblasts were cultured in 12-well plates and incubated for $96 \mathrm{~h}$ at $37^{\circ} \mathrm{C}$ in MEM medium with $0.4 \mathrm{mmol} / \mathrm{L} \mathrm{L-carnitine,} 4 \mathrm{~g} / \mathrm{L}$ bovine serum albumin (BSA) and $120 \mu \mathrm{mol} / \mathrm{L}$ of [U- $\left.{ }^{13} \mathrm{C}\right]$-palmitate. After $96 \mathrm{~h}$ incubation medium was collected. Cells were washed with PBS and harvested with 0.4 $\mathrm{mmol} / \mathrm{L} \mathrm{NaOH}$. Protein levels were determined using bicinchoninic acid (BCA) assay with human serum albumin (HSA) in $0.4 \mathrm{mmol} / \mathrm{L} \mathrm{NaOH}$ used as a standard. Collected medium was deproteinized using acetonitrile and subsequently butyl esters were formed. The dried butyl esters were dissolved in acetonitrile and analyzed using tandem mass spectrometry. ${ }^{2} \mathrm{H}_{3}$-C3-carnitine, ${ }^{2} \mathrm{H}_{3}-\mathrm{C} 8$ carnitine, ${ }^{2} \mathrm{H}_{3}-\mathrm{C} 16$ carnitine were used as internal standards. For intracellular acylcarnitine profiling in the hiPSC-CMs, cells were cultured on RPMI 1640 plus insulin-containing B27 supplement for $96 \mathrm{~h}$. Cells 
were harvested with trypsin and cell pellets were extracted in acetonitrile with internal standards and measured on high performance liquid chromatography mass spectrometry by a Q-ExactiveTM mass spectrometer (Thermo Scientific).

\subsection{Generation and Maintenance of hiPSC Lines}

Dermal fibroblasts of two VLCADD patients were expanded and reprogrammed to hiPSCs by Sendai virus (CytoTune-iPS 2.0 Sendai Reprogramming Kit, \#A16517,Thermo Fisher, Waltham, MA, USA, according to manufacturer's instructions) -mediated delivery of genes, specifically OCT4, KLF4, $c-M Y C, S O X 2$, as previously described [46]. Fibroblasts from both patients and from a healthy control (a 22 year old healthy male) were reprogrammed to generate hiPSC lines (iVLCADD1 from Patient 1 , iVLCADD2 from Patient 2 [46] and iCTRL [47,48] (Figure S1)). The experiments were performed on one hiPSC line per individual (named iVLCADD1 and iVLCADD2) after verification of their karyotype integrity (Figure S1A), pluripotency genes expression (Figure S1B,C) and differentiation potential (Figure S1D,E). A previously characterized skin fibroblasts-derived hiPSC line was used as control (iCTRL) [48]. All lines were maintained in mTeSR1 chemically defined medium on matrigel coated cell culture vessels and passaged using $0.5 \mathrm{mmol} / \mathrm{L}$ EDTA.

\subsection{Differentiation of hiPSC Lines into Cardiomyocytes}

All lines were differentiated in the presence of RPMI medium (Gibco) supplemented with B27 (GIBCO), in absence of insulin from day 1 to day 7 (with the addition of the molecules described below) and in presence of insulin from day 8 to day 30. Differentiation started when the hiPSC cultures were 70-90\% confluent. Mesoderm was induced using CHIR99021, Activin A and BMP4, followed by Wnt-pathway inhibition by $5 \mu \mathrm{mol} / \mathrm{L}$ IWP4 (modified from Dambrot et al. [49]). Since these hiPSC lines appeared quite sensitive to the growth factors treatment as published (i.e., high mortality) we applied a specific modulation of the concentration and duration of treatment with these factors to generate the optimal conditions for their highest survival and differentiation into cardiomyocytes. Concentrations of these factors and duration of their stimulation were adapted in a hiPSC line-specific manner as indicated. For the control hiPSC line (iCTRL): $1.5 \mu \mathrm{mol} / \mathrm{L}$ CHIR99021, $20 \mathrm{ng} / \mathrm{mL}$ Activin A, $20 \mathrm{ng} / \mathrm{mL}$ BMP4 on day 1, no factors on day 2, IWP4 on day 3-5; for the iVLCADD1 line: $1.5 \mu \mathrm{mol} / \mathrm{L}$ CHIR99021, $10 \mathrm{ng} / \mathrm{mL}$ Activin A, $10 \mathrm{ng} / \mathrm{mL}$ BMP4 on day 1, no factors on day 2, $2.5 \mu \mathrm{mol} / \mathrm{L} \mathrm{IWP4}$ on day 3-4; for the iVLCADD2 line: $1.5 \mu \mathrm{mol} / \mathrm{L}$ CHIR99021,10 ng/mL Activin A, $15 \mathrm{ng} / \mathrm{mL}$ BMP4 on day1-3, no factors on day 4, $2.5 \mu \mathrm{mol} / \mathrm{L}$ IWP4 on day 5-7. The differentiating hiPSC cultures of all lines were enriched for cardiomyocytes through a previously described metabolic selection [50], by culturing the cells from day 31 to day 36 in glucose-depleted RPMI medium supplemented with $4 \mathrm{mmol} / \mathrm{L}$ sodium lactate.

\subsection{Preparation of hiPSC-CM for Electrophysiology}

Lactate-treated hiPSC-CM cultures were detached from the culture vessels (usually 12 wells of a 24-well tissue culture plate) using $5 \times$ TrypLE Select Enzyme (Gibco) in PBS for a few minutes at $37^{\circ} \mathrm{C}$. The cells were collected in a $15 \mathrm{~mL}$ tube and washed once with PBS to dilute away the enzyme, spun-down at $150 \times g$ for $5 \mathrm{~min}$. To obtain a dissociation to single cells, the pellet was carefully resuspended in $1 \mathrm{~mL}$ Low- $-\mathrm{Ca}^{2+}$ Tyrode solution (composition as described by Meijer van Putten et al. [51]) and incubated at room temperature for $10 \mathrm{~min}$. Subsequently, Liberase (Roche Chemicals) and Elastase (Serva) were added at a final concentration of $20 \mu \mathrm{g} / \mathrm{mL}$ and $0.94 \mu \mathrm{g} / \mathrm{mL}$, respectively, as well as $500 \mu \mathrm{L}$ RPMI medium containing 2\% B27 supplement. The cells were then incubated overnight (for 10-12 h) at $37{ }^{\circ} \mathrm{C}$ in presence of $5 \% \mathrm{CO}_{2}$. After the incubation the cells suspension was diluted to $6 \mathrm{~mL}$ with RPMI medium containing $2 \%$ B27 supplement and centrifugated at $160 \times g$ for $5 \mathrm{~min}$. The pellet was gently resuspended in $600 \mu \mathrm{L}$ RPMI medium containing $2 \%$ B27 supplement and $50 \mathrm{U} / \mathrm{mL}$ penicillin and $50 \mu \mathrm{g} / \mathrm{mL}$ streptomycin and seeded on matrigel-coated glass coverslips lying inside wells of a 24 well plate. Presence of small groups of cells in addition to single cells were tolerated to avoid an excessively harsh dissociation. The seeded cells were let 
recover at $37^{\circ} \mathrm{C}$ in presence of $5 \% \mathrm{CO}_{2}$ for 8-10 days, with medium change every other day, then subject to electrophysiological analysis. Stock solution of resveratrol (Sigma, St. Louis, USA) and etomoxir (Sigma, St. Louis, USA) were prepared in dimethyl sulfoxide (DMSO) and diluted in culture medium to a final concentration of $50 \mu \mathrm{mol} / \mathrm{L}$ resveratrol or $100 \mu \mathrm{mol} / \mathrm{L}$ etomoxir $48 \mathrm{~h}$ prior to electrophysiological measurement.

\subsection{Cellular Electrophysiology in hiPSC-CMs}

Data acquisition. Action potentials (APs) were measured using the amphotericin-perforated patch-clamp technique and an Axopatch 200B amplifier (Molecular Devices, Sunnyvale, CA, USA). Voltage control, data acquisition and analysis were realized with custom software. Pipettes (resistance 2-3 M $\Omega$ ) were pulled from borosilicate glass capillaries (Harvard Apparatus, UK) using a custom-made microelectrode puller. Cell membrane capacitance $(\mathrm{Cm})$ was calculated [51] and potentials were corrected for the calculated liquid junction potential [52]. Signals were low-pass-filtered with a cut-off of $5 \mathrm{kHz}$ and digitized at $40 \mathrm{kHz}$.

AP measurements. AP were recorded at $36 \pm 0.2{ }^{\circ} \mathrm{C}$ using an extracellular, Tyrode solution containing (in mmol/l): $140 \mathrm{NaCl}, 5.4 \mathrm{KCl}, 1.8 \mathrm{CaCl}_{2}, 1 \mathrm{MgCl}_{2}, 5.5$ glucose, 5 HEPES, pH 7.4 $(\mathrm{NaOH})$. Pipettes were filled with solution containing (in mmol/L): $125 \mathrm{~K}$-gluc, $20 \mathrm{KCl}, 5 \mathrm{NaCl}, 0.44$ amphotericin-B, 10 HEPES, pH 7.2 (KOH). Typically, hiPSC-CMs have a small or even complete lack of the inward rectifying potassium current (IK1) [51]. Consequently, hiPSC-CMs have a depolarized maximal diastolic potential (MDP) and are frequently spontaneously active [53]. We overcome this limitation by injection of an in silico $\mathrm{I}_{\mathrm{K} 1}$ with kinetics of Kir2.1 channels through dynamic clamp, as previously described in detail [51]. Here, we used an amount of $2 \mathrm{pA} / \mathrm{pF} \mathrm{I}_{\mathrm{K} 1}$ peak outward current which resulted in quiescent hiPSC-CMs with close-to-physiological resting membrane potential. APs were elicited at $1-\mathrm{Hz}$ by 3-ms, $\sim 1.2 \times$ threshold current pulses through the patch pipette. Susceptibility to delayed afterdepolarization (DADs) was tested by applying a 3-Hz pacing episode (10-s) followed by an 8-s pause. After the pause, a single AP was evoked to test the inducibility of early afterdepolarizations (EADs). DADs were defined as depolarizations larger than $1 \mathrm{mV}$ during the 8-s pause. We analyzed $\mathrm{MDP}$, AP amplitude $\left(\mathrm{APA}_{\max }\right)$, AP plateau amplitude $\left(\mathrm{APA}_{\text {plateau }}\right.$; measured at $20 \mathrm{~ms}$ after initiation of the action potential upstroke), maximum $\mathrm{AP}$ upstroke velocity (Vmax) and AP duration at $20 \%, 50 \%$ and $90 \%$ repolarization $\left(\mathrm{APD}_{20}, \mathrm{APD}_{50}\right.$ and $\mathrm{APD}_{90}$, respectively). Parameters from 10 consecutive $\mathrm{APs}$ were averaged. Number of DADs of $>1 \mathrm{mV}$ was counted and averaged over 5 recording traces.

\subsection{Cytoplasmic Calcium Measurements}

Cells were prepared for the calcium measurements in a similar manner as described above for the electrophysiology measurements. Intracellular calcium transients were measured in the hiPSC-CMs at $37^{\circ} \mathrm{C}$ stimulated at $1 \mathrm{~Hz}$, using a fluorescent probe Indo- 1 as described previously [54].

\subsection{Statistical Analysis}

Data are presented as mean \pm standard error of the mean (SEM) for the electrophysiological data or as mean \pm standard deviation (SD) for the biochemical experiments. Statistical analysis was carried out with SigmaStat 3.5 software and Graphpad Prism 7. Normality and equal variance assumptions were tested with the Kolmogorov-Smirnov and the Levene median test, respectively. Two groups were compared with unpaired t-test or, in case of a failed normality and/or equal variance test, Mann-Whitney Rank Sum Test. More than 2 groups were compared using One-Way ANOVA followed by a Student-Newman-Keuls Method post hoc test. $p<0.05$ was defined as statistical significance. The specific statistical test used are indicated in each figure legend.

Supplementary Materials: Supplementary materials can be found at http://www.mdpi.com/1422-0067/21/7/2589/ s1. 
Author Contributions: R.H.H. and C.R.B. conceived the study. S.J.G.K., L.I., G.V., R.J.A.W., F.A.W., R.H.H., C.R.B. designed the study. S.J.G.K., J.C.B. and G.V. clinically evaluated patients. S.F. provided biochemical data. K.G., W.L., X.L. and Y.U. reprogrammed the fibroblasts to patient-specific iPSC lines and performed pluripotency characterization. I.M. contributed to characterization of the patient-specific hiPSC lines, maintained the hiPSC lines and differentiated them to cardiomyocytes S.J.G.K., J.C.B. and R.C.I.W. dissociated cells and performed biochemical experiments. A.B. performed calcium measurements. V.M.P. and A.O.V. performed electrophysiology measurements. S.J.G.K., R.C.I.W., I.M., A.O.V. and A.B. analyzed the data. S.J.G.K., I.M., A.O.V., R.H.H. and C.R.B. wrote the manuscript. All authors critically revised the manuscript and gave final approval of the version to be published. All authors have read and agreed to the published version of the manuscript.

Funding: This work was supported by an Innovation Impulse Grant 2016 from the Academic Medical Center, Amsterdam. Work in the Houtkooper group is financially supported by a VIDI grant from ZonMw (no. 91715305) and a grant from the Velux Stiftung (no. 1063). Work in the Bezzina group is supported by the Dutch Heart Foundation (CVON PREDICT2 project), the Netherlands Organization for Scientific Research (VICI fellowship, 016.150.610) and Fondation Leducq. Work in the Guan group is financially supported by the Free State of Saxony and the European Union EFRE (SAB project "PhänoKard") and by the DFG (GU595/3-1, IRTG2251). RC was supported by the Leducq Foundation (RHYTHM 16CVD 02).

Conflicts of Interest: The authors declare no conflict of interest.

\section{References}

1. Mathur, A.; Sims, H.F.; Gopalakrishnan, D.; Gibson, B.; Rinaldo, P.; Vockley, J.; Hug, G.; Strauss, A.W. Molecular heterogeneity in very-long-chain acyl-CoA dehydrogenase deficiency causing pediatric cardiomyopathy and sudden death. Circulation 1999, 99, 1337-1343. [CrossRef] [PubMed]

2. Knottnerus, S.J.G.; Bleeker, J.C.; Wust, R.C.I.; Ferdinandusse, S.; Ijist, L.; Wijburg, F.A.; Wanders, R.J.A.; Visser, G.; Houtkooper, R.H. Disorders of mitochondrial long-chain fatty acid oxidation and the carnitine shuttle. Rev. Endocr. Metab. Disord. 2018, 19, 93-106. [CrossRef] [PubMed]

3. Vianey-Saban, C.; Divry, P.; Brivet, M.; Nada, M.; Zabot, M.T.; Mathieu, M.; Roe, C. Mitochondrial very-long-chain acyl-coenzyme A dehydrogenase deficiency: Clinical characteristics and diagnostic considerations in 30 patients. Clin. Chim. Acta Int. J. Clin. Chem. 1998, 269, 43-62. [CrossRef]

4. Bonnet, D.; Martin, D.; Pascale De, L.; Villain, E.; Jouvet, P.; Rabier, D.; Brivet, M.; Saudubray, J.M. Arrhythmias and conduction defects as presenting symptoms of fatty acid oxidation disorders in children. Circulation 1999, 100, 2248-2253. [CrossRef] [PubMed]

5. Kluge, S.; Kuhnelt, P.; Block, A.; Merkel, M.; Gocht, A.; Lukacs, Z.; Kohlschutter, A.; Kreymann, G. A young woman with persistent hypoglycemia, rhabdomyolysis, and coma: Recognizing fatty acid oxidation defects in adults. Crit. Care Med. 2003, 31, 1273-1276. [CrossRef]

6. Yamamoto, A.; Nakamura, K.; Matsumoto, S.; Iwai, M.; Shigematsu, Y.; Tajima, G.; Tsumura, M.; Okada, S.; Mitsubuchi, H.; Endo, F. VLCAD deficiency in a patient who recovered from ventricular fibrillation, but died suddenly of a respiratory syncytial virus infection. Pediatrics Int. Official J. Jpn. Pediatric Soc. 2013, 55, 775-778. [CrossRef]

7. Bleeker, J.C.; Kok, I.L.; Ferdinandusse, S.; van der Pol, W.L.; Cuppen, I.; Bosch, A.M.; Langeveld, M.; Derks, T.G.J.; Williams, M.; de Vries, M.; et al. Impact of NBS for VLCAD deficiency on genetic, enzymatic and clinical outcomes. J. Inherit. Metab. Dis. 2019, 42, 414-423. [CrossRef]

8. Pena, L.D.; van Calcar, S.C.; Hansen, J.; Edick, M.J.; Walsh Vockley, C.; Leslie, N.; Cameron, C.; Mohsen, A.W.; Berry, S.A.; Arnold, G.L.; et al. Outcomes and genotype-phenotype correlations in 52 individuals with VLCAD deficiency diagnosed by NBS and enrolled in the IBEM-IS database. Mol. Genet. Metab. 2016. [CrossRef]

9. Xiong, D.; He, H.; James, J.; Tokunaga, C.; Powers, C.; Huang, Y.; Osinska, H.; Towbin, J.A.; Purevjav, E.; Balschi, J.A.; et al. Cardiac-specific VLCAD deficiency induces dilated cardiomyopathy and cold intolerance. Am. J. Physiol. Heart Circ. Physiol. 2014, 306, 326-338. [CrossRef]

10. Diekman, E.; de Sain-van der Velden, M.; Waterham, H.; Kluijtmans, L.; Schielen, P.; van Veen, E.B.; Ferdinandusse, S.; Wijburg, F.; Visser, G. The Newborn Screening Paradox: Sensitivity vs. Overdiagnosis in VLCAD Deficiency. JIMD Rep. 2016, 27, 101-106. [CrossRef]

11. Knabb, M.T.; Saffitz, J.E.; Corr, P.B.; Sobel, B.E. The dependence of electrophysiological derangements on accumulation of endogenous long-chain acyl carnitine in hypoxic neonatal rat myocytes. Circ. Res. 1986, 58, 230-240. [CrossRef] [PubMed] 
12. Corr, P.B.; Creer, M.H.; Yamada, K.A.; Saffitz, J.E.; Sobel, B.E. Prophylaxis of early ventricular fibrillation by inhibition of acylcarnitine accumulation. J. Clin. Investig. 1989, 83, 927-936. [CrossRef] [PubMed]

13. Meszaros, J.; Pappano, A.J. Electrophysiological effects of L-palmitoylcarnitine in single ventricular myocytes. Am. J. Physiol. 1990, 258, H931-H938. [CrossRef] [PubMed]

14. Wu, J.; Corr, P.B. Palmitoyl carnitine modifies sodium currents and induces transient inward current in ventricular myocytes. Am. J. Physiol. 1994, 266, H1034-H1046. [CrossRef] [PubMed]

15. Wu, J.; Corr, P.B. Palmitoylcarnitine increases $\left[\mathrm{Na}^{+}\right]_{\mathrm{i}}$ and initiates transient inward current in adult ventricular myocytes. Am. J. Physiol. 1995, 268, H2405-H2417. [CrossRef] [PubMed]

16. Yamada, K.A.; Kanter, E.M.; Newatia, A. Long-Chain Acylcarnitine Induces $\mathrm{Ca}^{2+}$ Efflux from the Sarcoplasmic Reticulum. J. Cardiovasc. Pharmacol. 2000, 36, 14-21. [CrossRef]

17. Pei, Z.; Xiao, Y.; Meng, J.; Hudmon, A.; Cummins, T.R. Cardiac sodium channel palmitoylation regulates channel availability and myocyte excitability with implications for arrhythmia generation. Nat. Commun. 2016, 7, 12035. [CrossRef]

18. Yamada, K.A.; McHowat, J.; Yan, G.X.; Donahue, K.; Peirick, J.; Kleber, A.G.; Corr, P.B. Cellular uncoupling induced by accumulation of long-chain acylcarnitine during ischemia. Circ. Res. 1994, 74, 83-95. [CrossRef]

19. Hoffman, B.F.; Rosen, M.R. Cellular mechanisms for cardiac arrhythmias. Circ. Res. 1981, 49, 1-15. [CrossRef]

20. Devalla, H.D.; Gelinas, R.; Aburawi, E.H.; Beqqali, A.; Goyette, P.; Freund, C.; Chaix, M.A.; Tadros, R.; Jiang, H.; Le Bechec, A.; et al. TECRL, a new life-threatening inherited arrhythmia gene associated with overlapping clinical features of both LQTS and CPVT. EMBO Mol. Med. 2016, 8, 1390-1408. [CrossRef]

21. Bastin, J.; Lopes-Costa, A.; Djouadi, F. Exposure to resveratrol triggers pharmacological correction of fatty acid utilization in human fatty acid oxidation-deficient fibroblasts. Hum. Mol. Genet. 2011, 20, 2048-2057. [CrossRef] [PubMed]

22. Houtkooper, R.H.; Pirinen, E.; Auwerx, J. Sirtuins as regulators of metabolism and healthspan. Nat. Rev. Mol. Cell Biol. 2012, 13, 225-238. [CrossRef] [PubMed]

23. Aires, V.; Delmas, D.; Le Bachelier, C.; Latruffe, N.; Schlemmer, D.; Benoist, J.F.; Djouadi, F.; Bastin, J. Stilbenes and resveratrol metabolites improve mitochondrial fatty acid oxidation defects in human fibroblasts. Orphanet J. Rare Dis. 2014, 9, 79. [CrossRef]

24. Lopaschuk, G.D.; Wall, S.R.; Olley, P.M.; Davies, N.J. Etomoxir, a carnitine palmitoyltransferase I inhibitor, protects hearts from fatty acid-induced ischemic injury independent of changes in long chain acylcarnitine. Circ. Res. 1988, 63, 1036-1043. [CrossRef] [PubMed]

25. Wanders, R.J.; Ruiter, J.P.; Ijist, L.; Waterham, H.R.; Houten, S.M. The enzymology of mitochondrial fatty acid beta-oxidation and its application to follow-up analysis of positive neonatal screening results. J. Inherit. Metab. Dis. 2010, 33, 479-494. [CrossRef]

26. Casini, S.; Verkerk, A.O.; Remme, C.A. Human iPSC-Derived Cardiomyocytes for Investigation of Disease Mechanisms and Therapeutic Strategies in Inherited Arrhythmia Syndromes: Strengths and Limitations. Cardiovasc. Drugs Ther. 2017, 31, 325-344. [CrossRef]

27. Verkerk, A.O.; Veldkamp, M.W.; Bouman, L.N.; van Ginneken, A.C. Calcium-activated Cl- current contributes to delayed afterdepolarizations in single Purkinje and ventricular myocytes. Circulation 2000, 101, 2639-2644. [CrossRef]

28. Ferro, F.; Ouille, A.; Tran, T.A.; Fontanaud, P.; Bois, P.; Babuty, D.; Labarthe, F.; Le Guennec, J.Y. Long-chain acylcarnitines regulate the hERG channel. PLoS ONE 2012, 7, e41686. [CrossRef]

29. Zhang, L.P.; Yin, J.X.; Liu, Z.; Zhang, Y.; Wang, Q.S.; Zhao, J. Effect of resveratrol on L-type calcium current in rat ventricular myocytes. Acta Pharmacol. Sin. 2006, 27, 179-183. [CrossRef]

30. Wu, J.; Corr, P.B. Influence of long-chain acylcarnitines on voltage-dependent calcium current in adult ventricular myocytes. Am. J. Physiol. 1992, 263, H410-H417. [CrossRef]

31. Janse, M.J.; D'Alnoncourt, C.N. Reflections on reentry and focal activity. Am. J. Cardiol. 1987, 60, $21 \mathrm{f}-26 \mathrm{f}$. [CrossRef]

32. Keating, M.T.; Sanguinetti, M.C. Molecular and Cellular Mechanisms of Cardiac Arrhythmias. Cell 2001, 104, 569-580. [CrossRef]

33. Wit, A.L. Afterdepolarizations and triggered activity as a mechanism for clinical arrhythmias. Pacing Clin. Electrophysiol. 2018, 41, 883-896. [CrossRef] 
34. Diekman, E.F.; Ferdinandusse, S.; van der Pol, L.; Waterham, H.R.; Ruiter, J.P.; Ijlst, L.; Wanders, R.J.; Houten, S.M.; Wijburg, F.A.; Blank, A.C.; et al. Fatty acid oxidation flux predicts the clinical severity of VLCAD deficiency. Genet. Med. Off. J. Am. Coll. Med Genet. 2015, 17, 989-994. [CrossRef] [PubMed]

35. Chen, I.Y.; Matsa, E.; Wu, J.C. Induced pluripotent stem cells: At the heart of cardiovascular precision medicine. Nat. Rev. Cardiol. 2016, 13, 333-349. [CrossRef] [PubMed]

36. Molkentin, J.D.; Lu, J.-R.; Antos, C.L.; Markham, B.; Richardson, J.; Robbins, J.; Grant, S.R.; Olson, E.N. A Calcineurin-Dependent Transcriptional Pathway for Cardiac Hypertrophy. Cell 1998, 93, 215-228. [CrossRef]

37. Sussman, M.A.; Lim, H.W.; Gude, N.; Taigen, T.; Olson, E.N.; Robbins, J.; Colbert, M.C.; Gualberto, A.; Wieczorek, D.F.; Molkentin, J.D. Prevention of Cardiac Hypertrophy in Mice by Calcineurin Inhibition. Science 1998, 281, 1690. [CrossRef] [PubMed]

38. Wallace, C.H.; Baczko, I.; Jones, L.; Fercho, M.; Light, P.E. Inhibition of cardiac voltage-gated sodium channels by grape polyphenols. Br. J. Pharmacol. 2006, 149, 657-665. [CrossRef]

39. Gillingham, M.B.; Scott, B.; Elliott, D.; Harding, C.O. Metabolic control during exercise with and without medium-chain triglycerides (MCT) in children with long-chain 3-hydroxy acyl-CoA dehydrogenase (LCHAD) or trifunctional protein (TFP) deficiency. Mol. Genet. Metab. 2006, 89, 58-63. [CrossRef]

40. Tucci, S.; Behringer, S.; Spiekerkoetter, U. De novo fatty acid biosynthesis and elongation in very long-chain acyl-CoA dehydrogenase-deficient mice supplemented with odd or even medium-chain fatty acids. FEBS J. 2015, 282, 4242-4253. [CrossRef]

41. Jones, P.M.; Butt, Y.; Messmer, B.; Boriak, R.; Bennett, M.J. Medium-chain fatty acids undergo elongation before beta-oxidation in fibroblasts. Biochem. Biophys. Res. Commun. 2006, 346, 193-197. [CrossRef] [PubMed]

42. Carnielli, V.P.; Sulkers, E.J.; Moretti, C.; Wattimena, J.L.; van Goudoever, J.B.; Degenhart, H.J.; Zacchello, F.; Sauer, P.J. Conversion of octanoic acid into long-chain saturated fatty acids in premature infants fed a formula containing medium-chain triglycerides. Metabolism 1994, 43, 1287-1292. [CrossRef]

43. Primassin, S.; Tucci, S.; Herebian, D.; Seibt, A.; Hoffmann, L.; ter Veld, F.; Spiekerkoetter, U. Pre-exercise medium-chain triglyceride application prevents acylcarnitine accumulation in skeletal muscle from very-long-chain acyl-CoA-dehydrogenase-deficient mice. J. Inherit. Metab. Dis. 2010, 33, 237-246. [CrossRef] [PubMed]

44. Streckfuss-Bomeke, K.; Wolf, F.; Azizian, A.; Stauske, M.; Tiburcy, M.; Wagner, S.; Hubscher, D.; Dressel, R.; Chen, S.; Jende, J.; et al. Comparative study of human-induced pluripotent stem cells derived from bone marrow cells, hair keratinocytes, and skin fibroblasts. Eur. Heart J. 2013, 34, 2618-2629. [CrossRef]

45. Olpin, S.E.; Manning, N.J.; Pollitt, R.J.; Clarke, S. Improved detection of long-chain fatty acid oxidation defects in intact cells using [9,10-3H] oleic acid. J. Inherit. Metab. Dis. 1997, 20, 415-419. [CrossRef]

46. Ben Jehuda, R.; Eisen, B.; Shemer, Y.; Mekies, L.N.; Szantai, A.; Reiter, I.; Cui, H.; Guan, K.; Haron-Khun, S.; Freimark, D.; et al. CRISPR correction of the PRKAG2 gene mutation in the patient's induced pluripotent stem cell-derived cardiomyocytes eliminates electrophysiological and structural abnormalities. Heart Rhythm. 2018, 15, 267-276. [CrossRef]

47. Takahashi, K.; Tanabe, K.; Ohnuki, M.; Narita, M.; Ichisaka, T.; Tomoda, K.; Yamanaka, S. Induction of pluripotent stem cells from adult human fibroblasts by defined factors. Cell 2007, 131, 861-872. [CrossRef]

48. Dudek, J.; Cheng, I.F.; Balleininger, M.; Vaz, F.M.; Streckfuss-Bomeke, K.; Hubscher, D.; Vukotic, M.; Wanders, R.J.; Rehling, P.; Guan, K. Cardiolipin deficiency affects respiratory chain function and organization in an induced pluripotent stem cell model of Barth syndrome. Stem Cell Res. 2013, 11, 806-819. [CrossRef]

49. Dambrot, C.; Braam, S.R.; Tertoolen, L.G.; Birket, M.; Atsma, D.E.; Mummery, C.L. Serum supplemented culture medium masks hypertrophic phenotypes in human pluripotent stem cell derived cardiomyocytes. J. Cell. Mol. Med. 2014, 18, 1509-1518. [CrossRef]

50. Tohyama, S.; Hattori, F.; Sano, M.; Hishiki, T.; Nagahata, Y.; Matsuura, T.; Hashimoto, H.; Suzuki, T.; Yamashita, H.; Satoh, Y.; et al. Distinct metabolic flow enables large-scale purification of mouse and human pluripotent stem cell-derived cardiomyocytes. Cell Stem Cell 2013, 12, 127-137. [CrossRef]

51. Meijer van Putten, R.M.; Mengarelli, I.; Guan, K.; Zegers, J.G.; van Ginneken, A.C.; Verkerk, A.O.; Wilders, R. Ion channelopathies in human induced pluripotent stem cell derived cardiomyocytes: A dynamic clamp study with virtual IK1. Front. Physiol. 2015, 6, 7. [CrossRef] [PubMed]

52. Barry, P.H.; Lynch, J.W. Liquid junction potentials and small cell effects in patch-clamp analysis. J. Membr. Biol. 1991, 121, 101-117. [CrossRef] [PubMed] 
53. Hoekstra, M.; Mummery, C.L.; Wilde, A.A.; Bezzina, C.R.; Verkerk, A.O. Induced pluripotent stem cell derived cardiomyocytes as models for cardiac arrhythmias. Front. Physiol. 2012, 3, 346. [CrossRef] [PubMed]

54. Baartscheer, A.; Schumacher, C.A.; Fiolet, J.W. Cytoplasmic sodium, calcium and free energy change of the $\mathrm{Na}^{+} / \mathrm{Ca}^{2+}$-exchanger in rat ventricular myocytes. J. Mol. Cell. Cardiol. 1998, 30, 2437-2447. [CrossRef] [PubMed] 